\title{
COEXISTING DEPRESSION AND ANXIETY: CLASSIFICATION AND TREATMENT
}

\author{
Kulhan T. ${ }^{1,}$, Ondrejka I. ${ }^{1}$,Ordaz J.A. ${ }^{3}$, Snircova E. ${ }^{1,2}$, Nosalova G. ${ }^{1}$ \\ ${ }^{1}$ Department of Pharmacology, Jessenius Faculty of Medicine, Comenius University, Martin \\ ${ }^{2}$ Clinic of Psychiatry, Jessenius Faculty of Medicine, Comenius University, University Hospital, Martin \\ ${ }^{3}$ Clinic of Anesthesiology and Intensive Medicine, Jessenius Faculty of Medicine, Comenius University, University \\ Hospital, Martin, Slovakia
}

\begin{abstract}
A b s t r a t
Despite of the fact, that comorbidity of depression and anxiety is a frequent condition in clinical practice, current psychiatric classification systems (according to WHO-ICD 10 and according to APA-DSM IV-TR) are not taking this reality into account sufficiently. The concept of anxious depression is very important for clinical practice. Recommended guidelines and algorithms of treatment based on evidence based medicine (EBM), established mainly on randomized controlled trials are designed separately for depression and separately for anxious disorders. This presents very often a significant complication in clinical practice. The aim of this article was to bring the concept of anxious depression to closer attention with highlighting of possible therapeutic approaches.
\end{abstract}

Key words: depression, anxiety, classification, treatment

\section{INTRODUCTION}

Depression is a common mental disorder that affects about 121 million people worldwide, it is the leading cause of disability as measured by YLDs (years lived with disability) and is already the 2nd cause of DALYs (Disability Adjusted Life Years) in the age category 15-44 years for both sexes combined. Depression, at its worst, is associated with the loss of about 850,000 lives every year (1).

The prevalence of depression in Europe is estimated in $10.05 \%$ for women and $6.61 \%$ for men (2).

The definition of depression varies according to the classification system. According to the DSM-IV the illness defined as depressive disorders include major depressive disorder, dysthymia and depressive disorders not otherwise specified (3). And according to the International Statistical Classification of Diseases and Related Health Problems 10th Revision (ICD-10) is part of mood (affective) disorders in which is included bipolar affective disorder, current episode of depression; depressive episode, recurrent depressive disorder, cyclothymia, dysthymia and unspecified mood disorders (4).

Illness currently defined in DSM-IV as anxiety disorders includes panic disorder, obsessive compulsive disorder, posttraumatic stress disorder (PTSD), generalized anxiety disorder, social phobia or social anxiety disorder and agoraphobia (3). The ICD-10 classifies as phobic anxiety disorders: agoraphobia, social phobias, specific (isolated) phobias, other phobic disorders and unspecified. Other anxiety disorders in the ICD-10 includes: panic disorder, generalized anxiety disorder, and mixed anxiety and depressive disorder, other mixed anxiety disorders, other specified anxiety disorders and unspecified; obsessive-compulsive disorder, reaction to severe stress and adjustment disorders. General anxiety disorder is one of the most frequent (up to 10\%) of all mental disorders seen in primary care and they are high utilizers of all health care resources (5).

Ad dress for correspond e n c e:

Tomas Kulhan, M.D., Clinic of Psychiatry, Jessenius Faculty of Medicine and University Hospital Martin, Kollarova

2, 03659 Martin, Slovak Republic 


\section{COMORBIDITY}

Comorbidity of depression with other psychiatric disorders is the rule, not the exception, in clinical practice. Often patients with depression have a comorbid anxiety disorder, and the converse is true as well (6). Studies of adults suggest that both disorders often share common risk factors and that similar interventions may be effective (7).

Closely related to anxiety and depression is also somatization and somatoform disorders, where characteristics are multiple physical complaints that are not caused by or cannot fully be explained by a known nonpsychiatric medical condition. The physical complaints include pain in different areas and symptoms other than pain involving gastrointestinal tract, sexual/reproductive system, respiratory system, dermatologic and neurologic system. Stekel refers to it also in terms of organ speech of the mind, meaning the organic expression of mental processes. It is as if the organs of the body were translating into a physiopathological language the mental troubles of the individual (8).

At least a third of patients with somatoform disorders have comorbid anxiety or depressive disorders, whereas depression and anxiety co-occur with one another up to $50 \%$ of the time (9).

\section{PREVALENCE}

Several studies focused on comorbidity of depression and anxiety were made.

According to a study by Aartijan et al. (7), high levels of comorbidity were found among major depressive disorder and anxiety disorders (47.5\% of those with major depressive disorder had comorbid anxiety disorders; $26.1 \%$ of those with anxiety disorders had comorbid major depressive disorder). In a comparison of specific anxiety disorders, comorbid major depressive disorder was more common in panic disorder and Obsessive-Compulsive Disorder - OCD (50.0\% and $44.4 \%$, respectively) than in generalized anxiety disorder and social phobia (30.3\% and $25.0 \%$, respectively) (7).

Another study shows that $75 \%$ of depressed patients had comorbid anxiety, somatization or both; $57 \%$ of the patients with anxiety had comorbid depression, somatization; or both and $54 \%$ of the patients with somatization had comorbid depression, anxiety or both (9).

Although it has been repeatedly confirmed that concurrent occurrence of depressive and anxious symptomatology is often, the concept of anxious depression is not sufficiently incorporated into the classification and appropriate therapeutic recommendations of this comorbidity are lacking.

Additionally, the probability that depression will be comorbid with well defined anxiety syndromes is nearly twice as high as probability that depression will be comorbid with alcohol dependence (10).

\section{IMPORTANCE}

Each of these disorders is associated with substantial functional impairment, increased disability days and elevated health care costs, but the contribution of the commonalities of depression, anxiety and somatization to functional impairment substantially exceeded the contribution of their independent parts (10).

Individuals who suffer from both anxiety and depression appear to experience a more chronic course and more impairment of social and occupational functioning than individuals with either anxiety or depression alone (10).

Comorbidity of depression and anxiety should be considered for several reasons:

a) Bigger severity: The group with anxiety disorders plus major depressive disorder may represent those with a more severe disorder (7). 
b) Worse outcome: If not treated properly, patients with comorbid anxiety and depression have a worse outcome than patients with either illness alone. Thus, the most effective treatment requires attention to symptoms of both anxiety and depression (6).

c) Withdrawal from treatment and decreased response to treatment: Several studies have found that greater severity of anxiety symptoms is associated with an increased risk of withdrawal from treatment, a decreased response to acute antidepressant treatment, and a longer time to both response and remission (11).

d) Risk of recurrence: High pretreatment levels of anxiety symptoms increase not only the risk of non-response in acute treatment of late-life depression but also the risk of recurrence of the disorder in the first 2 years after response to treatment (11).

e) Higher number of symptoms: In addition, patients with chronic medical illness and comorbid depression or anxiety compared to those with chronic medical illness alone report significantly higher numbers of medical symptoms (12).

\section{Syndrome overlap explanation:}

1. Depression, anxiety and somatization are defined by partially overlapping diagnostic criteria (3), which increase the likelihood of co-occurrence.

2. One syndrome might act as risk factor for the development of the other syndromes. For example, it has been shown that anxiety is a risk factor for the development of depression (13).

3. Depression, anxiety and somatization might emerge from shared psychosocial and biological diatheses. In fact, there is evidence to suggest that many of the phenomena of the somatoform disorders are caused by clustering of psychiatric syndromes or their incomplete or atypical manifestations and a low sensation threshold (14).

\section{CLASSIFICATION}

The first category of the newer classification system, which is currently used in DSM-IV as well, is a diagnosis of major depressive disorder in addition to a diagnosis of panic disorder, social phobia, generalized anxiety disorder, obsessive-compulsive disorder, or posttraumatic stress disorder.

The second category is a diagnosis of mixed anxiety-depressive disorder, which can only be made if the criteria for any other anxiety or mood disorder are not currently met (10). This diagnosis reflects the current, usually long lasting presence of mild depressive and mild anxiety symptomatology. Etiologically, it is neurotic, stress-induced impairment and thus, the diagnosis cannot be used for major depression-depressive episode with anxiety.

All or- nothing type of classification of mental disorders is a rather arbitrary process, which may hinder achieving better understanding of anxiety and depression (7).

The large syndrome overlap suggests that there may be a broader underlying construct consisting of depressive, anxiety, and somatic symptoms, that is, one disorder with three related types of symptoms rather than three distinct disorders. Indeed, some authors argue for a broader underlying disorder, labeled general neurotic syndrome or negative affect syndrome (9).

\section{TREATMENT}

Therapeutic approaches to comorbid depression and anxiety include pharmacologic and psychosocial options.

The pharmacologic choices include benzodiazepines, azapirones (non-benzodiazepine anxiolitics e.g. buspirone), antidepressants or combined treatment. Antidepressants are a safer alternative to benzodiazepines in patients who might become dependent on the latter agents (7). 
It is generally believed that all antidepressant medications are approximately equally effective for the treatment of depression. However, these medications are not equally effective in the treatment of anxiety disorders. For example, the serotonin reuptake inhibitors are more effective than tricyclic antidepressants in the treatment of OCD, and monoamine oxidase inhibitors may be more effective than tricyclic antidepressants in treating social phobia.

Several antidepressant medications are indicated for the treatment of certain anxiety disorders, whereas other antidepressants have not been consistently shown to also be effective in treating anxiety disorders. Certain medications have acquired a reputation of being more or less anxiogenic or anxiolytic than others and pharmaceutical companies have developed promotional campaigns suggesting that some medications are particularly well suited for treating depressed patients with anxious features. While knowledge of the presence of an anxiety disorder in a depressed patient might influence the choice of medication prescribed, there are, in fact, few data to support suggestions that depressed patients with anxious features respond differently to the range of antidepressant medications (16).

Determination of the pattern of the depression (e.g. endogenous or melancholic, atypical, nonendogenous, agitated, retarded) can have important clinical implications. For example, atypical depression, which is characterized by hypersomnia / hyperphagia, weight gain, reversed diurnal variation (worse in the evening), reactive mood, and heightened interpersonal sensitivity, is preferentially responsive to the monoamine oxidase inhibitors (MAOIs), and possibly selective serotonin reuptake inhibitors (SSRIs), that to the tricyclic antidepressants (TCAs).

It is important to note though, that using of the MAOIs may have possible limitations resulting from their known adverse effects. This fact is predominantly referred to their older generation of nonselective and irreversible IMAOs (e.g fenelzine, izokarboxazide), where diet free of tyramine was necessary and hypertension crisis occurred together with their hepatotoxic potential.

The newer generation of selective IMAOs from the end of the 1980-ties: reversible type A (moclobemide) and irreversible type B (selegiline), are considered as a safer alternative (15).

In evaluation of anxiety symptoms, many of the same considerations regarding depression apply, such as a review of other causes for anxiety symptoms. Determination of the presence of a specific anxiety disorder is important and can have implications for the choice of treatment also (19). Even when subdiagnostic anxiety symptoms exist with major depression, targeting the coexisting anxiety syndrome may be important.

Coexisting major depression and panic disorder: Panic disorder is characterized as an episodic paroxysmal anxiety. The efficacy of the available SSRIs (citalopram, escitalopram, fluoxetine, paroxetine, sertraline and fluvoxamine) in the acute treatment and long term maintenance of patients with major depression has been documented in several research trials (19). The SSRIs have also been used successfully in the treatment of uncomplicated panic disorder and are increasingly used as the first-line treatment for panic disorder (20). It is plausible, therefore, that these agents may be useful in the treatment of panic disorder with comorbid depression. The use of these agents is favoured especially in cases in which other antidepressants are not well tolerated (17). Worth mentioning is surely also group of SNRI agents (e.g. venlafaxine), which shows promising results in the treatment of coexisting major depression and panic disorder.

Coexisting major depression and social phobia: Social phobia is a disorder characterized by fear and avoidance of situations in which an individual may be scrutinized by other and fears that he or she will be embarrassed of humiliated (3). Social phobia is the third most common lifetime mental disorder in the general U.S. population (13.1\%), following major depression (17.1\%) and alcohol dependence (14.1\%) (21). Major depression is frequently associated with social phobia, and conversely, social phobia is often complicated by major depression. From a differential diagnostic perspective, it is important to discriminate social phobia from the social withdrawal associated with depressive illness (18).

Treatment of the patients with social phobia is particularly important because of the significant impairment in functioning, social isolation, and depression that accompany it (22). 
Based on the information currently available, patients with comorbid major depression and social phobia should probably receive antidepressant treatment. For patients suffering from comorbid atypical depression, MAOIs may be the optimal treatment. The SSRIs have been established as effective for patients with major depression (22), and, like the MAOIs, also appear to be more effective than the TCAs for patients with atypical depression (23). There is also growing evidence that SSRIs are beneficial in the treatment of social phobia. Thus, these agents may also have a therapeutic role in patients with coexisting major depression and social phobia, Because of the inconvenience associated with MAOI treatment, a trial with 1 or more SSRIs is a reasonable first treatment for major depression coexisting with social phobia. If the SSRIs in ineffective or not tolerated, after a washout of 2 weeks (or 5 weeks for fluoxetine), an MAOI such as phenelzine or tranylclypromine may be used. Addition of a high/potency benzodiazepines may be considered, if necessary (17).

Coexisting major depression and obsessive-compulsive disorder: A relatively small group of patients presenting with major depression also have OCD, but there is a strong association of OCD with depression. Rasmussen et al. (24), observed that $80 \%$ of a 44 OCD patient sample had a lifetime history of major depression; 30\% met criteria for major depression at the time of evaluation.

The SSRIs are effective in the treatment of both depression and OCD (25). The first systematic study of comorbid major depression and OCD was reported by Hoehn-Saric et al. (26), in that study, which compared the SSRI sertraline, the TCA desipramine, and placebo, the SSRI was superior to desipramine and placebo in alleviating both depression and OCD; (although, there is evidence that clomipramine, another agent form the TCA group, has anti-obsessional effect and should be also considered as one of the possible treatment option). Generally, SSRIs appear to be superior in alleviating concurrent depressive and OCD symptoms; other anti-obsessionals have not yet been studied in rigorous fashion (17).

Coexisting major depression and posttraumatic stress disorder: Davidson et al. (27), evaluated lifetime diagnoses of 44 World War II and Vietnam veterans and found that 59\% met lifetime criteria for major depression. Despite high rates of depression in posttraumatic stress disorder( PTSD) patients, antidepressants and psychotherapeutic techniques targeted at reducing depressive symptoms were less effective in depressed patients with PTSD than in patients with depression alone (28).

Based on clinical neuroendocrine challenge response differences between patients with major depression with and without PTSD, Southwick et al. (28), suggested that depressive symptoms in PTSD may not simply be manifestation of a concurrent major depressive episode, but rather may reflect either secondary or "nontypical" depression.

Since etiology of comorbid depression accompanying PTSD may differ significantly from the primary major depression, patients with PTSD probably require different, individualized treatment interventions (17).

Coexisting major depression and generalized anxiety disorder (GAD): Sanderson et al. (28), reported that over half of a sample of approximately 100 patients presenting for treatment of major depression or for dysthymia also had anxiety disorders; approximately $20 \%$ of each group had GAD. Other study evaluated 109 patients with GAD and found that $42 \%$ had experienced at least 1 major depressive disorder during their lifetime, with the first episode typically beginning shortly after the onset of GAD (30).

Benzodiazepines have traditionally been the treatment of choice in patients with GAD. However, coexisting depression and GAD raise questions regarding monotherapy with benzodiazepines in this patient subgroup. There is some evidence in the literature suggesting that the triazolobenzodiazepine alprazolam, may have specific antidepressant properties (31).

Based on the data, it appears that the TCAs or buspirone may have useful role in the treatment of GAD with prominent depressive symptomatology, in the same time, is suggested that patients with anxious depression respond well to the SSRIs and mirtazapine, NaSSA agent, which is noradrenergic and specifically serotonergic antidepressant with dual mechanism of effect (by increasing synthesis and releasing of noradrenalin and serotonin). These agents also appear to ameliorate depression-related anxiety of GAD associated with major depression (32). 
At this point, prior treatment responses, patient preference, and side effect profiles should be considered in the treatment decision process (17).

In the group of patients with anxious depression, where the intensity of symptoms is not severe and the risk of suicidal tendencies can be excluded, the combined therapy with psychopharmacs and procedures of psychosocial treatment (e.g. rehabilitation, cognitive behavioral therapy, interpersonal psychotherapy and other treatments) may be applied.

Additionally, also effect of other agents from different categories has been studied as a considerable form of pharmacologic treatment.

As effective appear: pregabaline, newer nonbenzodiazepine anxiolytic and GABA precursor; antihystaminics, e.g. hydroxyzine; buspirone; other groups of antidepressants - trazodone, with anxiolytic-sedative and sleep-inducing effect; agomelatine; SNRI (venlafaxine, duloxetine); some of the antipsychotics are proved to have anxiolytic and antidepressive effect and are often used in clinical practice in combination with $\mathrm{AD}$, also those from the first generation (basal), but because of adverse effects (anticholinergic, adrenolytic and other) they are used rather rarely and transiently in lower doses (e.g. chlorprothixen, levopromazine). More often are used the second generation antipsychotics in lower doses: benzamides - sulpiride, amisulpride, others like quetiapine, aripiprazole, risperidone, ziprasidone, olanzapine. If the cardiovascular symptoms of anxiety are prominent (tachycardia, palpitations, hypertension, but also tremor, hyperhydrosis, hyperventilation) beta-blockers may be effective; it is also considered anxiolytic - antidepressive effect of some of the antiepiletics, which could be possibly used as a therapy for patients with bipolar depression with anxious features (34).

From the natural substances effect of omega-3-fatty acids as adjuvant therapy of anxiety and mild depression is examined, as anxiolytics the possible effect have phytopharmaceuticals, e.g. Kava Kava, Galphimia glauca (Mexican natural medicine), Passionflower (passiflora), St. John's wort and others (34).

\section{CONCLUSION}

The presentation of patients with mixed symptoms of anxiety and depression is common in general medicine. It is estimated as the third most common ambulatory diagnosis in primary care practice (16).

The impairment and health care utilization associated with untreated mixed anxiety-depression is significant, and recognition and treatment of these patients are important (33).

It is clear that the presence of comorbid depression and anxiety can affect the course and treatment outcome. Identification of coexisting anxiety is very important and may determine the treatment of choice. In the same time, many patients with comorbid depression and anxiety require higher doses of medication and more intensive treatment to achieve remission than those without comorbid symptomatology (19).

Anxious depression as a diagnostic category does not exist in the DSM-IV; however, more study is needed to identify whether there is a residual category of patients with major depression with problematic anxiety symptoms.

The need for controlled studies comparing different agents that specifically include patients with coexisting anxiety and mood disorders is clear. Issues of comparative efficacy, treatment resistance, length of treatment, and relapse prevention remain areas requiring further investigation (19).

\section{REFERENCES}

1. World Health Organization. http://www.who.int.

2. Ayuso MJL, Vazquez BJL, Dowrick Ch et al. Depressive disorders in Europe: prevalence figures from the ODIN study. British Journal of Psychiatry 2001; 179: 308-16. 
3. American Psychiatric Association. Diagnostic and Statistical Manual of Mental Disorders, Fourth Edition, 2000 (DSM-IV-TR), Washington, DC: American Psychiatric Association; 2000.

4. The ICD-10 Classification of Mental and Behavioral disorders. Clinical descriptions and diagnostic guidelines. Geneva. Switzerland: World Health Organization; 1992.

5. King M, Nazareth I, Levy G, et al. Prevalence of common mental disorders in general practice attendees across Europe. British Journal of Psychiatry 2008; 192: 362-367.

6. Papaport MH. Prevalence, recognition and treatment of comorbid depression and anxiety. J Clin Psychiatry 2001; 62 (suppl 24): 6-10.

7. Aartjan T.F, Beekman, Beurs E, et al. Anxiety and Depression in Later Life: Co-Occurrence and communality of risk factors. Am J Psychiatry 2000; 157:89-95.

8. Campell RJ. Psychiatric dictionary. The definitive dictionary of psychiatry. Oxford. Ninth edition. 2009.

9. Bernd L, Spitzer RL, Williams JBW, et al. Depression, anxiety and somatization in primary care: syndrome overlap and functional impairment. General Hospital Psychiatry 2008; 30: 191-199

10. Muller HJ. Anxiety associated with comorbid depression. J Clin Psychiatry 2002; 63 (suppl 14): 22-26

11. Andreescu C, Lenze EJ, Dew MA, et al. Effect of comorbid anxiety on treatment response and relapse risk in late-life depression: controlled study. British Journal of Psychiatry 2007; 190: 344-349

12. Katon W, Eh L, Kroenke K. The association of depression and anxiety with medical symptom burden in patients with chronic medical illness. Gen Hosp Psychyiatry 2007; 29:147-55

13. Barlow Dh, Allen LB, Choate ML. Toward a unified treatment for emotional disorders. Behavior Therapy 2004; 35:205-30.

14. Mergl R, Seidscheck I, Allgaier AK, et al. Depressive, anxiety, and somatoform disorders in primary care: prevalence and recognition. Depress Anxiety 2007; 24 (3): 185-95 .

15. Bouček J, Pidrman V. Psychofarmaka v medicíně. Grada. Praha. 2005.

16. Zimmerman M, Dermut WM, Mattia JI. Frequency of anxiety disorders in psychiatric outpatients with major depressive disorder. Am J Psychiatry 2000; 157:1337-1340.

17. Lydiard RB, Mintzer B. Anxious depression. J Clin Psychiatry 1998; 59 (suppl 18): 10-17.

18. Rosenbaum Jf, Fava M, Nierenberg AA. The pharmacologic treatment of mood disorders. Psychiatr Clin North Am. Annual of Drug Therapy 1994; 1:17-50.

19. Fava M, Rush J. Difference in treatment outcome in outpatients with anxious versus nonanxious depression: A Star D Report. Am J Psychiatry 2008; 165: 342-351.

20. Nardi AE, Valença AM, Freire RC, et al. Psychopharmacotherapy of panic disorder: 8-week randomized trial with clonazepam and paroxetine. Braz J Med Biol Res. 2011; Apr 44 (4): 366-73.

21. Goes FS, McCusker MM, Bienvenu OJ, et al. Co-morbid anxiety disorders in bipolar disorder and major depression: familial aggregation and clinical characteristics of co-morbid panic disorder, social phobia, specific phobia and obsessive-compulsive disorder. Psychol Med 2011; Nov 21: 1-11.

22. Jonathan RT, Davidson, MD; Edna B, et al. Comprehensive cognitive behavioral therapy and placebo in generalized social phobia. Arch Gen Psychiatry 2004; 61:1005-1013.

23. Levitan RD, Jarrett RB, Schaffer M, et al. Treatment of atypical depression with cognitive therapy or phenelzine. Arch Gen Psychiatry 2000; 57: 1084.

24. Rasmussen SA, Eisen JL, Pato MT. Current issues in the pharmacologic management of obsessive compulsive disorder. J Clin Psychiatry 1993; 54 (suppl 6): 4-9.

25. Saric RH, Ninan P, Black DW, et al. Multicenter double-blind comparison of sertraline and desipramine for concurrent obsessive-compulsive and major depressive disorders. Arch Gen Psychiatry 2000; 57: 76 - 82.

26. Saric RH, Clary HW. Obsessive-complusive disorder with comorbid major depression: a comparison of sertraline and desipramine treatment. Eur Neuropsychopharmacol 1997; (suppl 2): 180.

27. Davidson JRT, Kudler HS, Saunders WB, et al. Symptom and morbidity patters in World War II and Vietnam veterans with post-traumatic stress disorder. Compr Psychiatry 1990; 31:1662-1670.

28. Southwick SM, Yehuda R, Giller EL. Characterization of depression in war-related posttraumatic stress disorder. Am J Psychiatry 1991; 148:179-183.

29. Sanderson WC, Beck AT, Beck J. Syndrome comorbidity in patients with major depression or dysthymia: prevalence and temporal relationships. Am J Psychiatry 1990; 147: 1025-1028.

30. Moffitt TE, Harrington HL, Caspi A, et al. Depression and generalized anxiety disorder: cumulative and sequential comorbidity in a birth cohort followed prospectively to age 32 Year. Arch Gen Psychiatry 2007; 64: $651-660$.

31. Klein E. The role of extended-release benzodiazepines in the treatment of anxiety: A risk-benefit evaluation with focus on extended-release alprazolam. J Clin Psychiatry 2002; 63(suppl 14): 27-33.

32. Goodwin RD, Gorma JM. Psychoparmacologic treatment of generalized anxiety disorder and the risk of major depression. Am J Psychiatry 2002; 159:1935-1937.

33. Crown WH, Finkelstein S, Berndt ER, et al. The impact of treatment-resistant depression on health care utilization and costs. J Clin Psychiatry 2002; 63 (11): 963-71.

34. Bazire S. Psychotropic drug directory 2012. The professionals pocket handbook and aide memoire. LloydReinhold Communication LLP, 2012. 